\title{
Long-term measurements of cosmic ray fluxes in the atmosphere
}

\section{Yuri Stozhkov, Vladimir Makhmutov, Nikolai Svirzhevsky, Galina Bazilevskaya,}

\section{Albina Svirzhevskaya, Mikhail Krainev, Valery Logachev}

P.N. Lebedev Physical Institute of the Russian Academy of Science

Leninsky Prospect, 53, 119991 Moscow, Russian Federation

stozhkov@fian.fiandns.mipt.ru

\section{Abstract}

The experimental data on galactic cosmic ray fluxes in the atmosphere are presented for five 11-year solar cycles from the 19-th solar cycle till the 24-th one. The cosmic ray data were obtained in the northern and southern polar atmospheres and in the northern atmosphere at the middle latitude. The analysis of monthly averaged of cosmic ray fluxes was made separately for positive and negative phases of 22-year solar magnetic cycles to get the evaluation of cosmic ray drift fluxes. Also, the relationship between cosmic ray fluxes at the altitudes of $(0.5-4.5) \mathrm{km}$ and global temperature in the boundary layer of the Earth's surface was analyzed. It was shown that the negative relationship exists between these values.

The 34th International Cosmic Ray Conference

30 July- 6 August, 2015

The Hague, The Netherlands 
1. Introduction

We present the data on cosmic ray fluxes in the atmosphere obtained at polar latitudes (northern polar latitude with the geomagnetic cutoff rigidity $R_{\mathrm{c}}=0.5 \mathrm{GV}$; southern one with $R_{\mathrm{c}}=$ $0.04 \mathrm{GV}$ ) and at the middle northern latitude with $R_{\mathrm{c}}=2.4 \mathrm{GV}$. The data have been obtained from the ground level up to the altitudes of (30-35) $\mathrm{km}$ and they cover the period from June 1957 to present time. Here we have used the monthly averaged data. Below is a brief description of the used measurement devices and the method of measurements.

To measure cosmic ray fluxes in the atmosphere the special radiosonde and devices on the ground to record radio signals from it were elaborated. The modern radiosonde is shown in Fig. 1. The Geiger tubes have been used to record charged particles. These tubes were manufactured in the USSR and now they are made in the Russian Federation. The rubber meteorological balloons are used to lift radiosondes up to $(30-35) \mathrm{km}$.

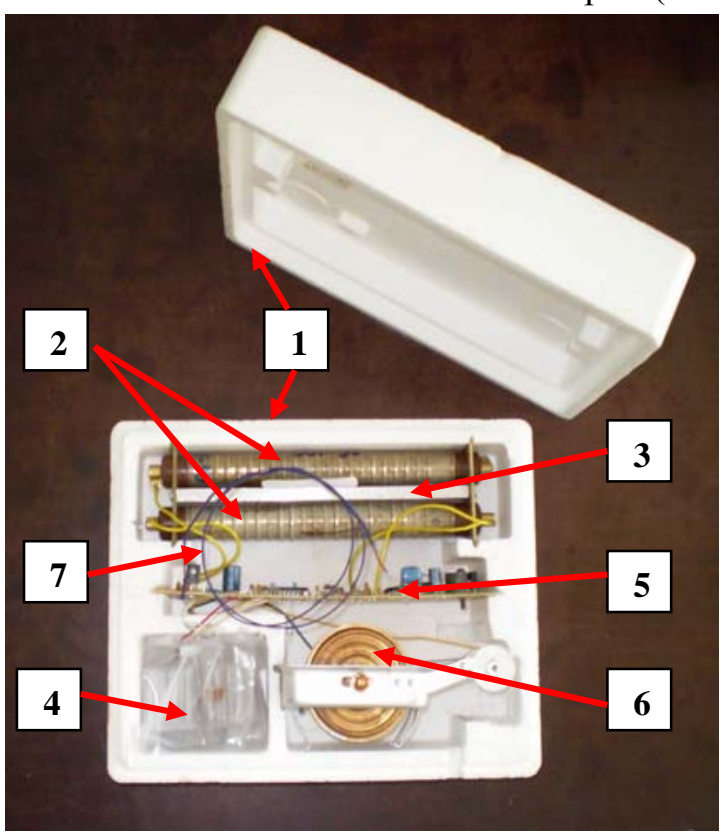

Fig.1. The modern standard radiosonde to measure charged particles in the Earth's atmosphere: 1 - foam plastic box; 2 - an upper Geiger tube of STS-6 type records global flux of charged particles, a telescope consisting of upper and bottom tubes with the $7 \mathrm{~mm}$ aluminium plate in-between (3) records vertical flux of charged particles; 4 - battery to power radiosound; 5 radioscheme with high voltage $(450 \mathrm{~V})$ and transmitter $(\sim 135 \mathrm{MHz})$; 6 atmospheric pressure sensor; 7 - antenna.

The radiosonde has three radio channels to transmit to the Earth the information on the crossing of upper single counter by a charged particle, on the crossing of telescope by a charged particle from vertical direction. Telescope consists of two single counters and aluminum 7-mm plate between them and it records charged particles from vertical directions. The geometrical factor of the single counter equals to $16 \mathrm{~cm}^{2}$ for isotropic flux of charged particles falling on it from the upper atmosphere. The geometrical factor for telescope is $18 \mathrm{~cm}^{2} \mathrm{sr}$ for isotropic flux of particles. The aluminum 7-mm plate absorbs all charged particles from radioactivity. Also, radiosonde transmits the information on the atmospheric pressure. Thus, the radiosonde transmits the information using 3 channels: pulses from single counter, from telescope and from atmospheric pressure sensor.

During 58 years of cosmic ray monitoring in the atmosphere about 85000 balloon launchings at the different latitudes from equatorial to polar ones were performed $[1,2]$. 


\section{Experimental data}

In Fig. 2 the monthly averaged cosmic ray fluxes $N_{\mathrm{m}}$ at maximum absorption curve in the atmosphere obtained at the different latitudes (with geomagnetic cutoff rigidity $R_{\mathrm{c}}=0.5 \mathrm{GV}, R_{\mathrm{c}}$ $=0.04 \mathrm{GV}$, and $R_{\mathrm{c}}=2.4 \mathrm{GV}$ ) are given.

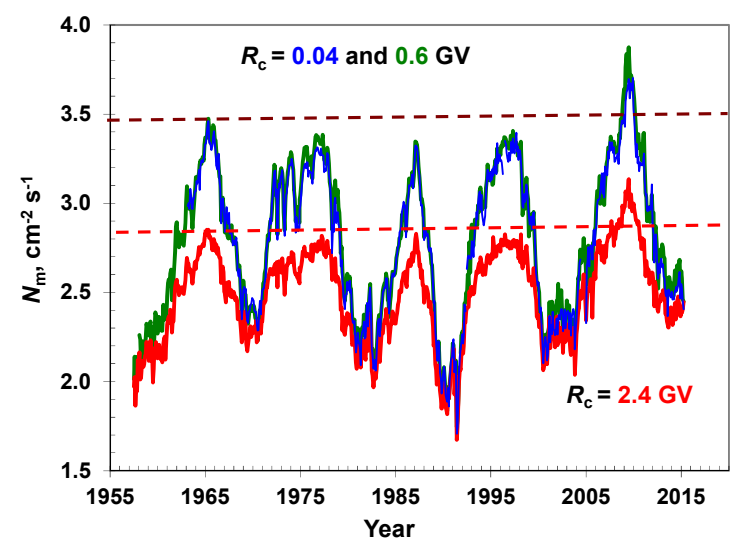

Fig. 2. Time profile of monthly averaged cosmic ray fluxes $N_{\mathrm{m}}$ at Pfotzer's maximum in the atmosphere at the northern latitude (Murmansk region, $R_{\mathrm{c}}=0.6 \mathrm{GV}$, green curve), the southern latitude (Antarctica, Mirny station, $R_{\mathrm{c}}=0.04 \mathrm{GV}$, blue curve), and the middle northern latitude (Moscow region, $R_{\mathrm{c}}=2.4 \mathrm{GV}$, red curve). Dashed brown and red lines mark the maximum of cosmic ray fluxes recorded in May, 1965.

As one can see from Fig. 2 we have the data covering five 11-year solar cycles (from maximum to maximum in solar activity) with rather large amplitudes $A>40 \%$. During all history of the regular observations of cosmic rays in the atmosphere the highest cosmic ray fluxes were recorded in the period of November, 2008 - February, 2010. The absolute maximum of $N_{\mathrm{m}}$ at the northern polar latitude was observed in July, 2009: $N_{\mathrm{m}}=3.874 \pm 0.042 \mathrm{~cm}^{-2} \mathrm{c}^{-1}$. It was higher than the cosmic ray flux observed in May, 1965 by $\sim 12 \%$. Note, that at the present time cosmic ray fluxes began to increase.

During 2008 - 2009 solar activity (sunspot number) and the strength of IMF were very low and it stimulated the cosmic ray increase. Several reasons could be responsible for the cosmic ray flux increase in 2009, namely: increase of the diffusion coefficient for cosmic ray particle propagation in the heliosphere or decrease of the dimension of modulation region size, or both cases are responsible for phenomena observed [3, 4].

Also in Fig. 2 the 22-year changes of galactic cosmic ray fluxes due to corresponding solar magnetic cycles are seen. During negative phases of 22-year solar magnetic cycles we observe sharp triangle forms of cosmic ray time profiles $(1960-1968 ; 1983-1988 ; 2006-2010)$ and we have flat curves during positive phases of solar magnetic cycles (1973 - 1978; 1993 - 1998). The drift effects are responsible for the observed different cosmic ray time profiles.

For comparison, the time profile of low energy cosmic ray flux is shown in Fig. 3. This flux is mainly due to the primary protons with energies of $(250-1500) \mathrm{MeV}$. Again, the 11year solar cycles are distinctly observed in this energy interval but their amplitudes are much higher than those given in Fig. 2. We see the 22-year cosmic ray flux changes but the differences between cosmic ray fluxes observed in negative and positive phases became smaller (except 2008 - 2009). In April, 2009 the low energy cosmic ray flux increased by $\sim 20 \%$ in comparison with May, 1965.

It is well known that cosmic ray fluxes at the Earth orbit are defined by solar activity level (sunspot number $R_{\mathrm{z}}$ ) and they are changed during 11-year solar cycle. The time profiles of the cosmic ray fluxes $N_{\mathrm{m}}$ and the values of $R_{\mathrm{z}}$ 


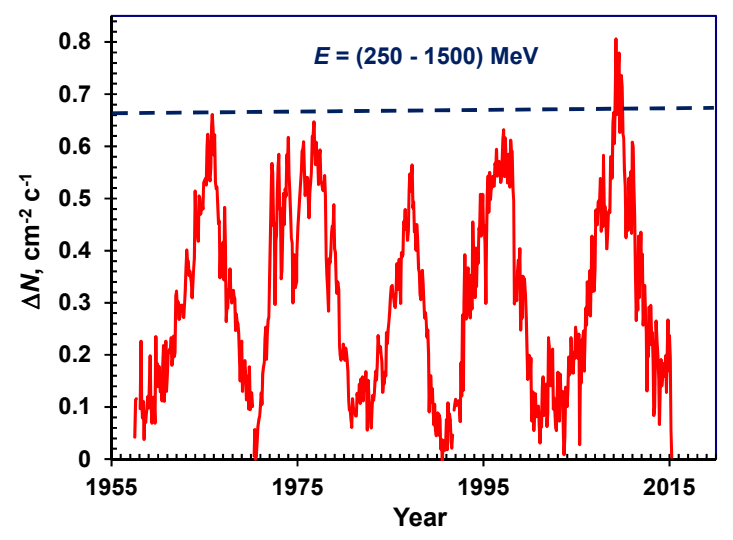

Fig. 3. Time profile of monthly averaged cosmic ray fluxes $\Delta N_{\mathrm{m}}$ at Pfotzer maximum in the atmosphere where $\Delta N_{\mathrm{m}}$ is the difference between $N_{\mathrm{m}}$ obtained at the northern polar latitude with $R_{\mathrm{c}}=0.5 \mathrm{GV}$ and $N_{\mathrm{m}}$ obtained at the northern middle latitude with $R_{\mathrm{c}}=2.4 \mathrm{GV}$ (see Fig. 2). These differences include the galactic cosmic ray particles with energies $(250-1500) \mathrm{MeV}$ falling on the top of the atmosphere. Dashed horizontal blue line shows maximum of cosmic ray flux $\Delta N_{\mathrm{m}}$ recorded in 1965 .

\section{Evaluation of drift fluxes of cosmic rays}

Now we will try to evaluate cosmic ray drift fluxes using the data presented in Fig. 1. Let us make some assumption: we accept that in average at the same solar acivity levels (the same values of sunspot number $R_{\mathrm{z}}$ ) in negative and positive phases of 22-year solar magnetic cycles the diffusion fluxes of cosmic rays are the same, $F_{\text {-dif }} \approx F_{+ \text {dif }}$, convective fluxes are the same, $F_{\text {-conv }} \approx F_{\text {+conv }}$ and drift fluxes are the same also, $F_{\text {-dr }} \approx F_{+\mathrm{dr}}$. But the latter have the opposite directions. In the positive phases drift fluxes are directed to the neutral heliospheric current sheet and in the negative phases they are directed from the current sheet. Under such assumptions if there is any differences of cosmic ray fluxes in positive and negative phases of solar magnetic cycles we can evaluate the value of drift fluxes: $F_{\mathrm{dr}}=\left(F_{+ \text {tot }}-F_{\text {-tot }}\right) / 2$, where $F_{\text {+tot }}=F_{+ \text {dif }}-F_{\text {+conv }}+F_{\text {+dr }}$ and $F_{\text {-tot }}=F_{\text {-dif }}-F_{\text {-conv }}-F_{\text {-dr. }}$.

We exclude from consideration the time intervals when the polar solar magnetic field inversion took place. To get the evaluations of drift fluxes let us take into account the delay time $\Delta t$ between solar activity $R_{\mathrm{z}}$ and cosmic ray fluxes $N_{\mathrm{m}}$.

The Table contains the time intervals under considerations, values of correlation coefficients $r$, and delay time $\Delta t$ between solar activity $R_{\mathrm{z}}$ and cosmic ray fluxes $N_{\mathrm{m}}$. During the time intervals under considerations the sunspot numbers were $20<R_{\mathrm{z}}<150$.

Table. The time intervals of negative and positive phases of 22-year solar cycles, correlation coefficients $r$, and delay time $\Delta t$ between solar activity $R_{\mathrm{z}}$ and cosmic ray fluxes $N_{\mathrm{m}}$.

\begin{tabular}{|c|c|c|c|c|c|}
\hline $\begin{array}{c}\text { Negative } \\
\text { phases }\end{array}$ & $r$ & $\begin{array}{c}\Delta t, \\
\text { month }\end{array}$ & $\begin{array}{c}\text { Positive } \\
\text { phase }\end{array}$ & $r$ & $\begin{array}{c}\Delta t, \\
\text { month }\end{array}$ \\
\hline $\begin{array}{c}01.1960- \\
02.1969\end{array}$ & -0.90 & 10 & $\begin{array}{c}01.1973- \\
10.1978\end{array}$ & -0.81 & 9 \\
\hline $\begin{array}{c}04.1982- \\
12.1989\end{array}$ & -0.90 & 9 & $\begin{array}{c}01.1993- \\
12.1998\end{array}$ & -0.82 & 5 \\
\hline $\begin{array}{c}01.2002- \\
12.2011\end{array}$ & -0.87 & 10 & & & \\
\hline
\end{tabular}

It is worth to note that in the positive phases the values of $r$ are lower than in the negative ones.

In Fig. 4 the relationship of $N_{\mathrm{m}}$ with $R_{\mathrm{z}}$ during positive and negative phases taking into account the delay time $\Delta t$ between them is shown. The $N_{\mathrm{m}}$ values were obtained at the northern polar atmosphere. 


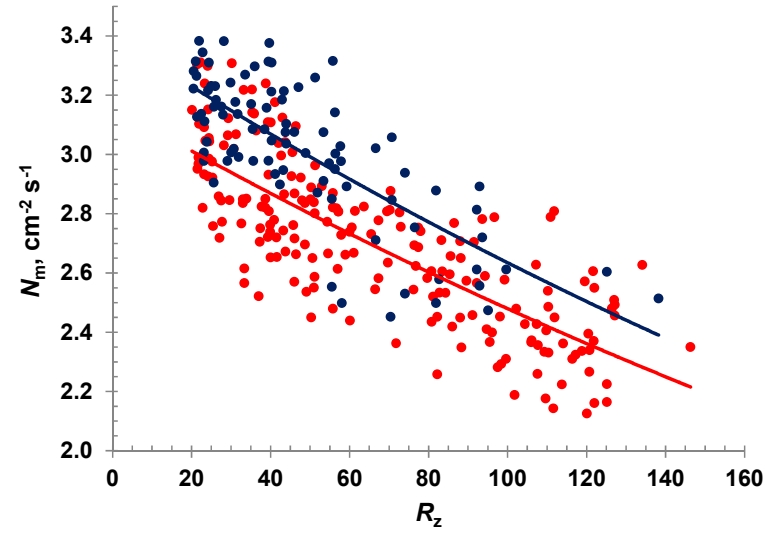

Fig. 4. The dependence of cosmic ray fluxes $N_{\mathrm{m}}$ from sunspot number $R_{\mathrm{z}}$. The values $N_{\mathrm{m}}$ were measured in the atmosphere at the northern polar latitude with $R_{\mathrm{c}}=0.5 \mathrm{GV}$. Red points belong to negative phases and blue points belong to positive ones. The solid curves are the approximations.

In spite of the large scattering of experimental points there is a separation of data obtained during positive phases (blue points) from the data obtained during negative ones (red points). The solid curves are the approximations: for negative phases (red points) it is $N_{\mathrm{m}}=$ 3.16 $\exp \left(-0.002 R_{\mathrm{z}}\right)$ with $r=-0.80$ and for positive phases (blue points) $N_{\mathrm{m}}=3.40 \cdot \exp \left(-0.003 R_{\mathrm{z}}\right)$ with $r=-0.76$.

The same relationship for the cosmic ray data obtained at the northern middle latitude with $R_{\mathrm{c}}=2.4 \mathrm{GV}$ is depicted in Fig. 5. Red points belong to negative phases and blue points belong to positive ones. The solid curves are the approximations. Again we see the separation between the experimental data for positive (blue points) and negative (red points) phases of 22year solar cycles. The approximations (solid curve) are described as: in negative phases $N_{\mathrm{m}}=$ $2.72 \cdot \exp \left(-0.002 R_{\mathrm{z}}\right)$ with $r=-0.77$ and in positive ones $N_{\mathrm{m}}=2.80 \cdot \exp \left(-0.001 R_{\mathrm{z}}\right)$ with $r=-0.73$.

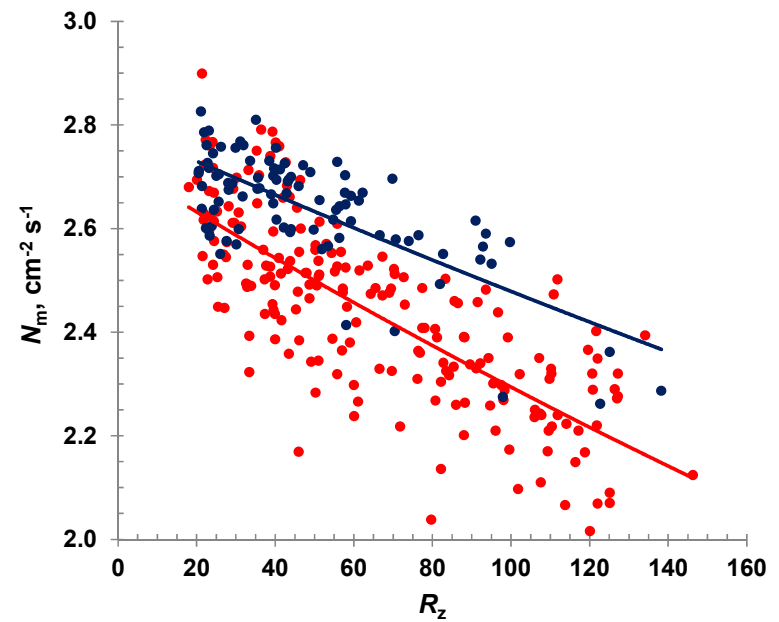

Fig. 5. The dependence of cosmic ray fluxes $N_{\mathrm{m}}$ from sunspot number $R_{\mathrm{z}}$. The values $N_{\mathrm{m}}$ were measured in the atmosphere at the northern middle latitude with $R_{\mathrm{c}}=2.4 \mathrm{GV}$. Red points belong to negative phases and blue points belong to positive ones. The solid curves are the approximations.

We assume that the differences between blue and red points in Figs. 4 and 5 are due to the drift fluxes. In the positive phases of the 22-year solar magnetic cycles the drift fluxes are directed to the neutral current sheet and in negative phases they are directed from the neutral current sheet. In the first case the cosmic ray fluxes observed at the Earth are increased and in the second one they are decreased.

From the data presented in Figs. 6 and 7 one can evaluate the value of drift flux of cosmic rays $F_{\mathrm{dr}}$ in the total counting rate $N_{\mathrm{m}}$ taking into account the suggestion given above. In our case the value of drift fluxes equals to $F_{\mathrm{dr}}=\left(F_{+ \text {tot }}-F_{\text {-tot }}\right) / 2$.

For the polar latitude $\left(R_{\mathrm{c}}=0.6 \mathrm{GV}\right)$ we obtain $F_{\mathrm{dr}}=\left[3.40 \cdot \exp \left(-0.003 R_{\mathrm{z}}\right)-3.16 \cdot \exp (-\right.$ $\left.\left.0.002 R_{\mathrm{z}}\right)\right] / 2 \approx 0.012+0.002 \cdot R_{\mathrm{z}} \mathrm{cm}^{-2} \mathrm{~s}^{-1}$ and for the middle latitude $\left(R_{\mathrm{c}}=2.4 \mathrm{GV}\right)$ we have $F_{\mathrm{dr}}=$ $\left[2.80 \cdot \exp \left(-0.001 R_{\mathrm{z}}\right)-2.72 \cdot \exp \left(-0.002 R_{\mathrm{z}}\right)\right] / 2 \approx 0.04+0.0013 \cdot R_{\mathrm{z}} \mathrm{cm}^{-2} \mathrm{~s}^{-1}$.

In the polar regions with low geomagnetic cutoff rigidity for $R_{\mathrm{Z}}=20$ the contribution of the drift flux in the total counting rate $N_{\mathrm{m}} \approx 3.3 \mathrm{~cm}^{-2} \mathrm{~s}^{-1}$ is $\left(F_{\mathrm{dr}} / N_{\mathrm{m}}\right) \approx(2-3) \%$. For $R_{\mathrm{z}}=100$ and 
$N_{\mathrm{m}} \approx 2.5 \mathrm{~cm}^{-2} \mathrm{~s}^{-1}$ drift fluxes are $\left(F_{\mathrm{dr}} / N_{\mathrm{m}}\right) \approx(8-9) \%$.

At the middle latitude for $R_{\mathrm{z}}=20$ and $N_{\mathrm{m}} \approx 2.7 \mathrm{~cm}^{-2} \mathrm{~s}^{-1}$ the contribution of the drift flux in the total counting rate is $\left(F_{\mathrm{dr}} / N_{\mathrm{m}}\right) \approx(1.5-2.5) \%$. For $R_{\mathrm{z}}=100$ we have $\left(F_{\mathrm{dr}} / N_{\mathrm{m}}\right) \approx(6-7) \%$.

Thus, the drift fluxes of cosmic rays are rather small in comparison with the diffusion and convective ones. It is valid for the low energy of particles, which we analyze. For example, the effective energy of particles at Pfotzer maximum at polar latitudes is about $(2-3) \mathrm{GeV}$ (note, that one of us, M. Krainev, has quite different opinion on the question considered in this section, see [5] and references therein).

\section{Influence of cosmic ray fluxes on global warming process}

Let us consider the important question about the cosmic ray role in the global warming process. Our analysis is based on the cosmic ray experimental data obtained at the northern middle latitude $\left(R_{\mathrm{c}}=2.4 \mathrm{GV}\right)$ at the altitudes from 0.5 up to $4.5 \mathrm{~km}$. We compare our data on cosmic ray fluxes in the atmosphere with the changes of global air temperature near the ground $\Delta T,{ }^{\circ} \mathrm{C}$. The changes of $\Delta T$ were calculated relative to the average temperature $T$ taken for the period of $1900-2000$ [6].

In the atmosphere at the altitudes under consideration the important processes of cloud formation take places. Many researches consider cosmic ray fluxes as a main factor responsible for cloud coverage over the Earth via ionization of air. For the first time the realationship between cosmic ray fluxes (equatorial neutron monitor data) and $\Delta T$ was found in [7]. But till present time hot discussion on the reality of this relationship take place. In the international CLOUD experiment at CERN charged particle fluxes is shown to be responsible for the increase of nucleation processes in air [8].

Here we show that the correlation of cosmic ray fluxes in the low atmosphere with $\Delta T$ values is observed. In Figs. 6 and 7 realtionship between the values of $N_{\mathrm{m}}$ (monthly averaged data) obtained at the northern middle latitude $\left(R_{\mathrm{c}}=2.4 \mathrm{GV}\right)$ in the low atmosphere at the altitudes $h=(0.5-2.2) \mathrm{km}$ and $h=(2.2-3.2) \mathrm{km}$ and the values of $\Delta T$ are given. The smoothing of $N_{\mathrm{m}}$ and $\Delta T$ values with the period $t_{\mathrm{sm}}=13$ months was made to decrease the data scattering. The periods of $01.1959-12.2003$ and $01.2011-03.2013$ were included in this analysis. The re-analysis of data for the period of $01.2004-12.2010$ is performed because for this period we had some problems with atmospheric pressure sensors.

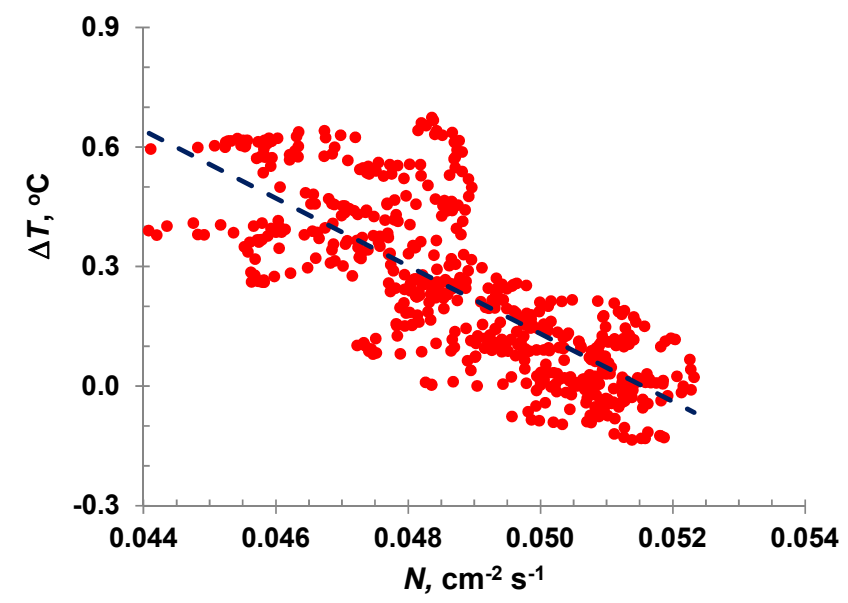

Fig. 6. The correlation between the values of $N_{\mathrm{m}}$ (monthly averaged data smoothed with the period $t_{\mathrm{m}}=13$ months) obtained at the northern middle latitude in the atmosphere at the altitudes of $h=(0.5-2.2) \mathrm{km}$ and the changes of the global air temperature near the Earth's surface $\Delta T,{ }^{\circ} \mathrm{C}$ [6]. Dashed line shows the linear correlation. The correlation coefficient equals to $r \approx-0.77$. 


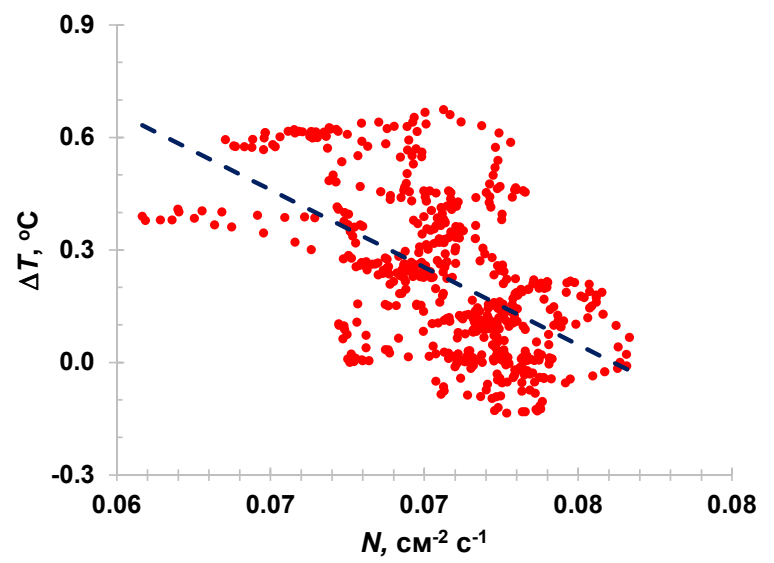

Fig. 7. The same as in Fig.6 but for the altitudes of $h=(2.2-3.2) \mathrm{km}$. The correlation coefficient equals to $r \approx-0.56$.

Also the correlation between the cosmic ray fluxes and the global temperature changes takes place if we take cosmic ray fluxes at the altitudes $h=(3.2-4.4) \mathrm{km}$. In this case the correlation coefficient is $r \approx-0.35$. At the higher altitudes the correlation becomes positive.

\section{Conclusion}

For the long-term period (1957 - present time) the unique experimental data on cosmic ray fluxes in the polar atmospheres and the middle northern one are presented.

The comparison of the data obtained during positive and negative phases of the several 22-year solar magnetic cycles shows that at the same solar activity level (the same sunspot number $R_{\mathrm{z}}$ ) there is the excess of cosmic ray particles during positive phases in comparison with negative ones. This excess is due to the drift fluxes. However, the value of this drift flux is low and equals to several percents $(\leq 10 \%)$ from the total cosmic ray flux observed.

The role of cosmic ray fluxes $N(h)$ at the low altitudes $h$ in the atmosphere in the global warming process is considered. The negative relationship between $N(h)$ in the low atmosphere $h=(0.5-4.5) \mathrm{km}$ and the global air temperature near the Earth's surface $\Delta T$ was found.

\section{Acknowledgments}

The authors thank for help the Russian Foundation for Basic Research (grant 14-02-00905) and the Program of the Presidium of the Russian Academy of Sciences "Fundamental Properties of Matter and Astrophysics".

\section{References}

[1] Stozhkov, Y.I., Svirzhevsky, N.S.,Bazilevskaya, G.A., Kvashnin, A.N., Makhmutov, V.S., Svirzhevskaya, A.K. Long-term (50 years) measurements of cosmic ray fluxes in the atmosphere. Advances in Space Research. 44 no. 10 (2009) 1124-1137. 
[2] Stozhkov, Y.I., Svirzhevsky, N.S.,Bazilevskaya, G.A., Krainev, M.B., Makhmutov, V.S., Svirzhevskaya, A.K., Logachev, V.I., Vasheyuk, E.V. Cosmic rays in the stratosphere in 2008 2010. Astrophys. Space Sci. Trans. 7(31) (2011) 379-382.

[3] Kalinin M.S., Bazilevskaya G.A., Krainev M.B., Svirzhevskaya A.K., Svirzhevsky N.S. On the description of the GCR intensity in the last three solar minima. 33-rd ICRC. (Astroparticle Physics Conference), Rio de Janeiro, 2013.

[4] M.S. Potgieter, E.E. Vos, M. Boezio, N. De Simone, V. Di Felice, V. Formato. Modulation of Galactic Protons in the Heliosphere. Solar Phys. (2014) 289:391-406. DOI 10.1007/s11207013-0324-6.

[5] M.B. Krainev, J. Kota, M.S. Potgieter. On causes and mechanisms of the long-term variations in the GCR characteristics. 34-th ICRC. Netherland, Hague, 2015, SH-Th-198.

[6] ftp://ftp.ncdc.noaa.gov/pub/data/anomalies/monthly.land_ocean.90S.90N. df_19012000mean.dat

[7] Svensmark H. and E. Friis-Christensen. Variation of cosmic ray flux and global cloud coverage - a missing link in solar-climate relationships. Journal of Atmospheric and Solar-Terrestrial Physics, 1977, 59(11), 1225-1232.

[8] Jasper Kirkby, Joachim Curtius, Joa o Almeida, et al. (CLOUD collaboration). Role of sulphuric acid, ammonia and galactic cosmic rays in atmospheric aerosol nucleation. Nature, 25 AUG US T 2011 | VOL 476 | N ATU RE | 429 - 433. doi:10.1038/nature10343. 